\title{
DESENVOLVIMENTO MOTOR DA MÃO DOMINANTE NOS MOVIMENTOS IDENTIFICADOS COMO RESPONSÁVEIS PELA PRODUÇÃO DA ESCRITA ${ }^{1}$
}

\author{
Liana Gonçalves Pontes Sodrê ${ }^{2}$
}

\begin{abstract}
Resumo
O presente trabalho é um estudo do desenvolvimento motor da mão dominante, nos 16 movimentos identificados como responsáveis pela reprodução da escrita manuscrita. Utilizou-se uma amostra de 256 crianças, de ambos os sexos, de duas categorias socioeconômicas, de três anos e meio a sete anos. As crianças foram distribuídas em 8 faixas etárias, em intervalos de seis meses, de modo que, em cada faixa etária, ficasse assegurado o mesmo número de meninos e meninas, das duas categorias socioeconômicas estudadas. Os resultados indicam que $50 \%$ das crianças da categoria socioeconômica alta foram capazes de reproduzir todos os movimentos e que, para as da categoria baixa, o índice de reprodução foi de $40 \%$. Quatro movimentos foram responsáveis pelas dificuldades de reprodução das crianças das duas categorias, o que permite identificá-los como movimentos críticos para esta faixa etária. As meninas, tal como em estudo piloto anterior, apresentaram maior freqüência de reprodução que os meninos, especialmente as da categoria socioeconômica alta. Estudos são sugeridos no propósito de acompanhar, em faixas etárias anteriores e iguais, o processo inicial de reprodução dos mesmos, mediante o estudo de casos exemplares.
\end{abstract}

\section{MOTOR DEVELOPMENT IN HANDEDNESS IN THE REPRODUCTION OF THE CURSIVE HANDWRITING}

\begin{abstract}
This is a study of the hand's development - left or right depending on handedness - in 16 movements identified as prior to and basic for the reproduction of cursive handwriting. It is based on a sample of 256 male and female children, between the ages of 3 and 7, from lower or upper midd1e to upper socio-economic backgrounds. The children were divided into 8 age groups, an interval of 6 months between each. In each age group there was the same number of boys and girls and of each socio-economic group studied. Results showed that $50 \%$ of the upper socio-econonúc groups were able to reproduce alI the movements against $40 \%$ of the other group. 4 movements were found as responsible for the difficulties of the children' s from both socioeconomics categories and these result allow to identify them as the most critical for these ages groups. The girls, as in an other pilot study, reproduce more frequently the movements than the boys, especially the girls of the upper socio-economic leveI. Several suggestions are made in given more attention to these aspects in children' s of the ages groups studied and in lower ages groups.
\end{abstract}

\section{Introdução}

O desenvolvimento motor, como outros aspectos, depende de fatores ambientais consoantes com as características físicas de cada criança. Acrescido a isso, o progresso no domínio desta, como de outras habilidades complexas, depende de uma seqüência encadeada de circunstâncias que favoreçam o domínio gradual de todos os aspectos que lhes são próprios.

\footnotetext{
${ }^{1}$ Dados extraídos da tese de Doutorado concluída em 1993.

${ }^{2}$ Professora Aposentada da Universidade Federal da Bahia.

Professora Adjunta do Campus X da UNEB.
} 
Para SCHILLER \& SCHILLER (1990), com referência ao desenvolvimento motor, as intervenções podem ser formais ou informais, dirigi das ou livres; o importante é que sejam oferecidas às crianças condições adequadas ao seu desenvolvimento. Ou seja, que estas condições sejam compatíveis com a idade das crianças, suas características e os aspectos do desenvolvimento que estão em pauta.

BOUCHER \& DOESCHER (1991) também defendem a idéia de que o desenvolvimento motor não depende de muita intervenção. Eles observaram dois grupos de crianças, que foram submetidos às condições estruturadas e não estruturadas de treinamento motor, respectivamente, e constataram melhoras no desenvolvimento motor de ambos, sem que fossem constatadas diferenças significativas entre eles.

A partir do primeiro ano de vida, quando as crianças começam a usar as mãos e a fala com mais autonomia para explorar o ambiente, HOL T (1977) destaca que a vida delas passa a se resumir a períodos de intensa atividade, alternados com períodos de sono. O desenvolvimento dessas crianças passa a ser determinado pelas possibilidades que lhes são oferecidas.

O referido autor considera os quatro primeiros anos de vida como o tempo necessário para a superação da dependência total do adulto e o início de uma autonomia com os cuidados pessoais e com os eventos do seu dia-a-dia. É neste sentido que ele realizou observações sistemáticas de crianças nesta faixa etária. Entre os diversos aspectos enfocados pelo seu estudo, destacaram-se, para a presente pesquisa, os aspectos que dizem respeito ao desenvolvimento motor manual, a saber: a) a criança de 4 anos já segura o lápis com o polegar e dois dos demais dedos (indicador e médio), controlando o lápis de tal modo que copia um círculo e uma cruz e esboça desenhos de formas humanas e casas; b) a criança de 5 anos já segura o lápis com maturidade e se diverte desenhando e pintando formas humanas e casas com clareza e com suas respectivas características.

Outro trabalho que também descreve, entre outros aspectos do desenvolvimento, o desempenho das crianças com relação à aquisição do domínio de instrumentos gráficos na reprodução de formas são os estudos de V AUGHAN \& LITT (1990). Eles observaram os comportamentos das crianças diante de objetos variados, inclusive colocando-os diante de instrumentos gráficos e papéis para que pudessem descrever a evolução da competência na reprodução de formas. Estavam interessados em coletar dados que descrevessem o desenvolvimento neurológico de crianças desde o nascimento até o $60^{\circ}$ mês de vida.

No que diz respeito ao desempenho na reprodução de formas, constataram o seguinte: aos 36 meses, as crianças mostraram-se aptas a copiar círculos e a imitar o desenho de uma cruz; com 48 meses, já copiavam uma cruz, um quadrado e já desenhavam um homem em duas a quatro partes; com 60 meses, mostraram-se aptas a desenhar triângulos.

Estes trabalhos acima citados, assim como os estudos clássicos de desenvolvimento, realizados por HURLOCK (1946), GESELL e AMATRUDA (1946), apontam evidências de que, após o terceiro ano de vida, o processo de desenvolvimento manual adquire o grau de mobilidade adequado aos refinados movimentos de preensão e manipulação de objetos. Esses estudos, mesmo dando indícios dos aspectos biológicos (pela representatividade da amostra observada) no processo de desenvolvimento, não deixam de enfatizar que não devem ser ignoradas as diferenças determinadas pelas variadas condições ambientais, sugerindo que os diferentes meios socioeconômicos podem favorecer uma apreciação melhor de como as possibilidades das habilidades exibidas ficam sob controle social. 
Os trabalhos de HOLT (1977) e de VAUGHAN \& LITT (1990) são exemplos de estudos de desenvolvimento que, entre outros aspectos, registram o início do envolvimento das crianças nas atividades gráficas na reprodução de traçados ou desenhos. Não se propuseram a descrever os movimentos manuais que são responsáveis por estes traçados.

É neste sentido que este trabalho se propõe a contribuir de modo mais efetivo. Os demais estudos descrevem os desempenhos envolvidos na reprodução da escrita ou do desenho infantil com as crianças manipulando instrumentos gráficos, como o lápis, sem descrever a possibilidade ou não destes movimentos sem a apreensão ou o uso destes instrumentos.

É uma proposta, portanto, de observação e registro dos 16 movimentos identificados como responsáveis pela reprodução dos componentes gráficos da escrita manuscrita. Eles foram identificados e definidos no estudo original (SODRÉ, 1982) a partir das observações efetuadas durante a reprodução de todos os numerais e de todas as letras maiúsculas e minúsculas da escrita manuscrita. Interessava na época, num processo dedutivo de descobertas, descrever que partes da mão e dedos eram responsáveis pela reprodução dos diversos componentes gráficos, quando da reprodução de todas as letras e numerais.

Considerávamos naquele momento (e ainda mantemos esta crença) que, especificamente com relação à escrita, se o homem atual já possui no seu repertório genético uma condição biológica (ossos e músculos) que lhe permite desenvolver esta habilidade e se é o ambiente social que favorece ou não o seu desenvolvimento, por conseguinte os estudos a ela pertinentes deveriam voltar-se para os aspectos significativos e funcionais indispensáveis à compreensão do processo.

Iniciamos uma série de estudos que tinham como ponto de partida a própria forma gráfica da escrita manuscrita cursiva do mundo ocidental. Já que nos interessava o domínio de sua reprodução, seguimos um caminho dedutivo, analisando primeiro a sua forma gráfica para obter uma descrição sintética de seus traçados ou, dito melhor, dos componentes de sua grafia. Em seguida, identificamos os movimentos manuais envolvidos na reprodução de cada um deles.

Esta pesquisa aqui relatada (SODRÉ, 1993), portanto, se propôs a observar movimentos da mão dominante, que tanto pode ser a esquerda como a direita, definida como a mais utilizada ou a única utilizada nas tarefas que necessitam apenas de uma das mãos. Mais especificamente, as observações foram limitadas aos movimentos do braço, antebraço, mão e dedos da mão dominante, na reprodução dos movimentos identificados como envolvidos na escrita, na seqüência que foi levantada e definida em estudos anteriores (SODRÉ, 1982 e 1985).

Este estudo não tem por objetivo a identificação de estágios ou fases, mas, sim, de descrever e analisar uma seqüência encadeada de movimentos. Esta seqüência pode ser relacionada com idades, ou melhor, faixas etárias, deixando claro que este referencial (faixa etária) será usado como indicador de maior possibilidade de quantidade e de variedade de experiências vivenciadas, do que como fatores delimitantes do desenvolvimento.

Uma outra preocupação deste trabalho é a de não definir expectativas para cada uma das faixas etárias em estudo. A proposta é de um estudo descritivo dos 16 movimentos reproduzidos pela mão dominante, usando os instrumentos, procedimentos e referenciais originários de trabalhos anteriores (SODRÉ, 1982 e 1985), para observação destes aspectos do repertório motor da mão dominante que se definiram como básicos para a atividade de escrita.

Uma segunda preocupação deste trabalho é verificar se as diferenças de gênero existem nestes aspectos do desenvolvimento, assim como foram constatadas diferenças de gênero em 
estudos de outros aspectos do desenvolvimento, como os de BUTTERWORTH, 1991;JONES\&GLENN, 1990;ENNIS, 1990;MEAD \& IGNICO, 1992; HACKER, 1992, entre outros, embora, como afirma JACKLIN (1989), a importância dessas diferenças no desenvolvimento não seja bem conhecida.

Para FAGOT (1985), a socialização cultural não se explica unicamente pela transmissão de papéis dos dois gêneros, do adulto para a criança, posto que ela envolve a compreensão e a interpretação dos papéis de cada gênero pelas crianças. A compreensão desses motivos é importante, visto que os estudos de WILLIAMS (1991) demonstraram que estes estereótipos de gênero já estão presentes nos pré-escolares e já se aproximam bastante das características adultas a partir da média infância.

KARAPETSAS \& VLACHOS (1992) observaram que as meninas, até os 12 anos, apresentam uma melhor organização visomotora que os meninos. Nas suas conclusões sugerem que os dois sexos vêem a figura de forma diferente e, com isso, levantaram indícios de que as organizações neuropsicológicas dos dois sexos, até esta idade, são diferentes. Estes resultados impõem ao presente estudo a necessidade de verificar se essas diferenças começam a se estabelecer no nível dos movimentos, como os que estão envolvidos na escrita cursiva.

Como terceiro aspecto a ser enfocado, destaca-se a importância de um estudo do possível efeito das diferenças socioeconômicas neste aspecto do desenvolvimento. Para HOLLE (1979) e BIJOU \& BAER (1980), as possibilidades de movimentos ficam sob controle social, já que é o ambiente que seleciona, a partir da estrutura biológica inerente a cada indivíduo, os movimentos que serão incorporados e mantidos no repertório de cada um.

Portanto, este estudo tem por propósito descrever o desenvolvimento da mão dominante, a partir da observação da reprodução dos 16 movimentos identificados como relacionados à atividade de escrita manuscrita, em crianças de sexo masculino e feminino, de 3 anos e meio a 7 anos (na faixa etária que precede a idade definida para a alfabetização - 7 anos), de origem socioeconômicas baixa e média-alta a alta, pelo uso dos procedimentos e critérios identificados e definidos em estudos anteriores.

\section{MÉTODO}

\section{Sujeitos}

São 256 crianças de duas categorias socioeconômicas distintas, na faixa etária de 3 anos e meio a 7 anos completos, de uma amostra significativa de crianças da cidade de Campinas; 128 crianças eram de origem socioeconômica média-alta e alta (das escolas particulares mais caras da cidade) e 128 de origem socioeconômica baixa (escolhidas aleatoriamente das instituições públicas de ensino ou creches da cidade que atendiam a esta população), sendo 50\% meninos e 50\% meninas nos dois grupos. As crianças dos dois grupos foram distribuídas em 8 grupos etários, em intervalos de 6 meses (estudo de cortes transversais), de modo a ficar assegurada a presença de meninos e meninas em todas as faixas etárias.

\section{Procedimento}

A coleta dos dados era realizada em um local isolado, livre de interferências que distraíssem as crianças, onde só permaneciam o pesquisador, a criança em observação e o auxiliar de pesquisa, 
que registrava a reprodução ou não dos movimentos. A mão dominante era identificada a partir de algumas perguntas sobre a mão que ela usava para pentear o cabelo, desenhar, etc. Em seguida, a criança era informada que deveria movimentar apenas aquela mão e dedos. O pesquisador se posicionava numa cadeira sem braços, à direita da cadeira da criança e a auxiliar à frente dos mesmos. Por fim, recebia a instrução de que deveria olhar para o pesquisador, observar o movimento por ele reproduzido e, em seguida, imitá-lo.

A estratégia utilizada, portanto, foi a de imitação de um modelo. O pesquisador executava cada um dos movimentos na ordem planejada, de modo que, em seguida, a criança o reproduzisse.

\section{Movimentos}

Os dois primeiros movimentos foram reproduzidos isoladamente, quais sejam: a) movimento 1 - flexão do braço em relação ao antebraço (a escrita é efetuada com o braço flexionado em relação ao antebraço); b) movimento 2 - pronação da mão (palma da mão voltada para baixo). Estes dois primeiros referem-se à postura básica para a escrita. Após a emissão de cada um desses movimentos pelo pesquisador, a criança era solicitada a imitá-lo.

Os demais 14 movimentos restantes foram solicitados em duplas. Isto porque observá-los em duplas mostrou-se eficiente para uma melhor avaliação de suas reproduções, pois a emissão de um movimento é favorecida pela emissão de seu oponente. Os 14 movimentos restantes foram reproduzidos na seguinte ordem:

movimentos 3 e 4 - flexão e extensão da mão

movimentos 5 e 6 - adução e abdução da mão

movimentos 7 e 8 - abdução e adução do polegar

movimentos 9 e 10 - flexão e extensão do polegar

movimentos 11 e 12 - abdução e adução dos dedos

movimentos 13 e 14 - flexão e extensão dos dedos

movimentos 15 e 16 - abdução e adução do indicador

\section{Folha de registro}

Uma folha de papel tamanho ofício com um cabeçalho para os dados de identificação e, logo abaixo, uma tabela de freqüência simples onde: no sentido vertical, dispôs-se o nome das crianças no canto esquerdo do papel e, ao longo do sentido horizontal, os números de identificação dos 16 movimentos. O registro limitava-se a um pequeno traço na casela correspondente à criança e ao movimento não reproduzido. Deste modo ficavam mais evidentes os movimentos mais críticos para esta população (explicando melhor: aqueles que elas não conseguiam reproduzir), pela freqüência de pequenos traços no sentido vertical, bem como as crianças com mais dificuldades, pela freqüência de pequenos traços no sentido horizontal.

\section{RESULTADOS}

O primeiro aspecto a ser destacado no estudo é a relação dos movimentos que foram reproduzidos por todas as crianças, quais sejam: 
movimento 1 - flexão do antebraço

movimento 2 - pronação da mão

movimentos 3 e 4 - flexão e extensão da mão

movimentos 11 e 12 - adução e abdução de todos os dedos

movimentos 13 e 14 - flexão e extensão de todos os dedos

Em seguida, os movimentos que foram reproduzidos por mais de 95\% da população estudada:

movimentos 7 e 8 - adução e abdução do polegar

movimentos 9 e 10 - flexão e extensão do polegar

Por conseguinte, os mais críticos para reprodução nesta faixa etária são os movimentos 5 e $\mathbf{6}$ (adução e abdução da mão) e os movimentos 15 e 16 (adução e abdução do indicador).

Quanto às diferenças de desempenhos no que diz respeito ao sexo e nível socioeconômico (NSE), os resultados podem ser mais bem analisados na Tabela 1. Nela está distribuído o porcentua1 das crianças, dos dois NSE, que reproduziram, ou não, os movimentos 5/6, 7/8, 9/10 e 15/16, de acordo com o sexo. Pode-se constatar que os movimentos mais críticos foram os movimentos 5 e 6, já que pouco mais da metade da população estudada (56,6\%) os reproduziu, seguido dos movimentos 15 e 16, com a reprodução de 73,8\%. Observa-se também que os desempenhos das crianças do NSE alto tiveram índices de reprodução maior que para as demais. Além disso, nesta categoria socioeconômica, os meninos apresentam índices de reprodução inferiores aos das meninas em todos os quatro pares de movimentos.

A aplicação do Qui Quadrado permitiu constatar a associação entre os movimentos 5 e 6 e a variável sexo. Mostrou-se significante ao nível de 5\% apenas para as crianças do NSE alto (53,1 \% meninos e $70,3 \%$ meninas). 
Tabela 1 - Porcentagem das crianças dos dois NSE que reproduziram, ou não, os movimentos 5/6, 7/8, 9/10 e 15/16, de acordo com o sexo.

\begin{tabular}{c|c|c|c|c|c|c|c}
\hline NSE/sexo & NSE A & NSE A & NSE A & NSE B & NSE B & NSE B & TOTAL \\
\hline Movimentos & Masc. & Fem. & Total & Masc. & Fem. & TOTAL & (n=256) \\
\hline Mov. 5/6 & & & & & & & \\
Reproduzem & 53,1 & 70,3 & 61,7 & 56,2 & 45,3 & 50,8 & 56,6 \\
Não rep. & 46,9 & 29,7 & 38,3 & 43,8 & 54,7 & 49,2 & 43,4 \\
\hline Mov.7/8 &. & & & & & & \\
Reproduzem & 96,9 & 100 & 98,4 & 95,3 & 100 & 97,7 & 98,0 \\
Não rep. & 3,1 & 0 & 1,6 & 4,7 & 0 & 2,3 & 2,0 \\
\hline Mov. 9/1 O & & & & & & & \\
& & & & & & & \\
Reproduzem & 96,9 & 98,4 & 97,7 & 100 & 100 & 100 & 98,8 \\
Não rep. & 3,1 & 1,6 & 2,3 & 0 & 0 & 0 & 1,2 \\
\hline Mov. 15/16 & & & & & & & \\
Reproduzem & 76,6 & 78,1 & 77,3 & 70,3 & 70,3 & 70,3 & 73,8 \\
Não rep. & 23,4 & 21,9 & 22,7 & 29,7 & 29,7 & 29,7 & 26,2 \\
\hline
\end{tabular}

Quanto à faixa etária, os dados estão distribuídos nas Tabelas 2 (NSE alto) e 3 (NSE baixo). Nelas estão distribuídos os resultados referentes às crianças dos dois grupos socioeconômicos estudados, de acordo com as 8 faixas etárias definidas para o estudo e o sexo, na reprodução dos movimentos 5/6, 7/8, 9/10 e 15/16. Na Tabela 2, verifica-se que os meninos do NSE alto reproduzem os movimentos 5 e 6 em índices muito baixos a partir dos 3 anos e meio (12,5\%). A reprodução destes movimentos por eles vai melhorando gradualmente, até atingir o porcentual de $75 \%$ aos 6 anos de idade. Quanto às meninas, de modo geral demonstram desempenhos melhores que os meninos. Entretanto, fica evidente a melhora gradual dos desempenhos em relação às diferentes faixas etárias, ou seja, quanto mais velhas, melhores os percentuais de reprodução.

Tabela 2 - Porcentagem das crianças do NSE alto, que reproduziram os movimentos 5/6, 7/8,9/10 e 15/16, de acordo com o sexo e a faixa etária $(\mathrm{n}=128)$.

\begin{tabular}{|c|c|c|c|c|c|c|c|c|}
\hline $\begin{array}{l}\text { Movimentos } \\
\text { Sexo } \\
\text { Faixa etária }\end{array}$ & $\begin{array}{c}\text { 5/6 } \\
\text { Masc. }\end{array}$ & $\begin{array}{r}5 / 6 \\
\text { fem. }\end{array}$ & $\begin{array}{c}\text { 7/8 } \\
\text { Masc. }\end{array}$ & $\begin{array}{r}7 / 8 \\
\text { fem. }\end{array}$ & $\begin{array}{c}\text { 9/10 } \\
\text { Masc. }\end{array}$ & $\begin{array}{l}\text { 9/10 } \\
\text { fem. }\end{array}$ & $\begin{array}{l}\text { 15/16 } \\
\text { Masc. }\end{array}$ & $\begin{array}{l}15 / 16 \\
\text { fem. }\end{array}$ \\
\hline 3 anos e m. & 12,5 & 75,0 & 87,5 & 100 & 87,5 & 87,5 & 62,5 & 50,0 \\
\hline 4 anos & 37,5 & 50,0 & 87,5 & 100 & 100 & 100 & 75,0 & 100 \\
\hline 4 anos e m. & 50,0 & 25,0 & 100 & 100 & 100 & 100 & 75,0 & 62,5 \\
\hline 5 anos & 50,0 & 75,0 & 100 & 100 & 100 & 100 & 75,0 & 87,5 \\
\hline 5 anos e m. & 50,0 & 75,0 & 100 & 100 & 87,5 & 100 & 62,5 & 75,0 \\
\hline 6 anos & 75,0 & 75,0 & 100 & 100 & 100 & 100 & 87,5 & 62,5 \\
\hline 6 anos e m. & 75,0 & 100 & 100 & 100 & 100 & 100 & 100 & 87,5 \\
\hline 7 anos & 75,0 & 87,5 & 100 & 100 & 100 & 100 & 75,0 & 100 \\
\hline
\end{tabular}


Quanto à Tabela 3, as crianças do NSE baixo evidenciam diferenças mais reduzidas. Excetuando os resultados melhores das meninas na reprodução dos movimentos 7 e 8 (100\% de reprodução em todas as faixas etárias), que é um resultado pouco representativo, principalmente porque os meninos saíram-se bem na reprodução deles, nos demais (os movimentos 5/6 e 15/ 16, que são mais críticos), em algumas faixas etárias, as meninas saem-se melhores, e em outras não.

O Qui Quadrado de tendência mostra uma associação entre as crianças do NSE alto, nas 8 faixas etárias, que reproduziram ou não os movimentos 5 e 6 (p aproximadamente igual a zero). Também para as crianças do NSE baixo o Qui Quadrado de tendência confirma a significância $(p=0,008)$. Isto indica que a reprodução destes movimentos melhora significativamente com a idade para as crianças dos dois níveis estudados.

Tabela 3 - Porcentagem das crianças do NSE baixo, que reproduziram os movimentos 5/6, 7/8, 9/10 e 15/16, de acordo com o sexo e a faixa etária $(n=128)$.

\begin{tabular}{|c|c|c|c|c|c|c|c|c|}
\hline $\begin{array}{l}\text { Movimentos } \\
\text { Sexo } \\
\text { Faixa etária }\end{array}$ & $\begin{array}{c}5 / 6 \\
\text { masco }\end{array}$ & $\begin{array}{l}5 / 6 \\
\text { fem. }\end{array}$ & $\begin{array}{c}7 / 8 \\
\text { masco }\end{array}$ & $\begin{array}{r}7 / 8 \\
\text { fem. }\end{array}$ & $\begin{array}{c}9 / 10 \\
\text { masco }\end{array}$ & $\begin{array}{l}9 / 10 \\
\text { fem. }\end{array}$ & $\begin{array}{r}15 / 16 \\
\text { masco }\end{array}$ & $\begin{array}{r}15 / 16 \\
\text { fem. }\end{array}$ \\
\hline 3 anos e m. & 37,5 & 37,5 & 75,0 & 100 & 100 & 100 & 50,0 & 62,5 \\
\hline 4 anos & 50,0 & 50,0 & 87,5 & 100 & 100 & 100 & 0,0 & 62,5 \\
\hline 4 anos e m. & 62,5 & 12,5 & 100 & 100 & 100 & 100 & 75,0 & 50,0 \\
\hline 5 anos & 37,5 & 25,0 & 100 & 100 & 100 & 100 & 62,5 & 50,0 \\
\hline 5 anos em. & 62,5 & 75,0 & 100 & 100 & 100 & 100 & 75,0 & 100 \\
\hline 6 anos & 87,5 & 50,0 & 100 & 100 & 100 & 100 & 87,5 & 75,0 \\
\hline 6 anos e m. & 62,5 & 62,5 & 100 & 100 & 100 & 100 & 87,5 & 75,0 \\
\hline 7 anos & 50,0 & 50,0 & 100 & 100 & 100 & 100 & 75,0 & 87,5 \\
\hline
\end{tabular}

Os resultados também evidenciam que, com relação à faixa etária, a significância nem sempre se deve às diferenças entre cada uma das oito faixas etárias, mas, sim, a uma diferença significativa entre grupos etários que variam a depender do NSE.

Com relação à reprodução dos movimentos 5/6, as crianças do NSE alto apresentam melhoras progressivas em três grupos etários: 3 anos e meio a 4 anos e meio (41,6\%); 5 a 6 anos (66,7\%); e 6 anos e meio a 7 anos (84,4\%). Já as do NSE baixo apresentaram melhoras mais abruptas, quais sejam: 3 anos e meio a 5 anos (39,1\%); e 5 anos e meio a 7 anos (62,5\%).

Para os movimentos 15/16, os diferentes grupos etários se evidenciam da seguinte forma: 3 anos e meio a 6 anos (73\%); e 6 anos e meio a 7 anos (90,6\%), para as do NSE alto. Por outro lado, para as do NSE baixo os dois grupos etários foram 3 anos e meio a 5 anos (57,9\%); e 5 anos e meio a 7 anos (82,8\%).

Concluindo, verifica-se que, com relação ao NSE, tanto na reprodução dos movimentos 5/6 como 15/16, as crianças apresentam patamares superiores no desempenho, com a progressão nas faixas etárias, sendo que, para o NSE alto, a melhora é gradual na reprodução dos movimentos 5/6 (3 grupos) e, para o NSE baixo, as melhoras são mais abruptas (2 grupos), tanto na reprodução dos movimentos 5/6 quanto nos movimentos 15/16. A faixa etária confirma-se, portanto, como uma variável significativa na reprodução dos movimentos estudados, porém em grupos etários que se 
diferenciam de acordo com o NSE.

\section{DISCUSSÃO}

O primeiro aspecto que merece ser discutido são as crianças que reproduziram todos os movimentos. As crianças do NSE alto reproduziram mais (24,6\%) do que as do NSE baixo (20,3\%). A diferença é pequena (4,3\%) e não é significativa, considerando as condições mais favoráveis das crianças da categoria alta, principalmente porque as diferenças são marcantes, tanto nas condições oferecidas pela família quanto nas oferecidas pelas instituições que freqüentam.

As diferenças de gênero, favorecendo as meninas, só foram confirmadas nas crianças das categorias mais altas e apenas na reprodução de 2 dos 16 movimentos. Isso leva a deduzir que, nas crianças do NSE alto, as diferenças individuais podem estar sobrepondo-se às diferenças socioculturais. Deste modo, as diferenças de gênero se impõem, e as meninas destacam-se dos meninos.

A faixa etária, portanto, foi a variável que se evidenciou como relevante para a população em estudo, na reprodução dos movimentos mais críticos para esta população. O percentual baixo de reprodução destes movimentos pode ser atribuído às faixas etárias iniciais.

A competência das crianças maiores deve estar refletindo não só a maior probabilidade de experiência que o tempo de vida propicia, mas também o atendimento especializado das professoras, que todas (mesmo as do NSE baixo) passam a receber a partir dos 5 anos. É um atendimento voltado para as atividades gráficas que antecedem a escrita, tais como desenhos, pinturas, recortes, etc.

O que precisa ser enfatizado com os resultados obtidos é que as 8 faixas etárias definidas para o estudo nem sempre mostraram variações marcadamente diferenciadas, ou seja, uma evolução gradual de melhora nos desempenhos. Parece que, a partir dos 5 anos e meio, as crianças passam a ser mais estimuladas, visto que passam a ser atendidas por professoras, na reprodução desses movimentos. Posto isso, os dados sugerem que, devido a um atendimento mais direcionado ou a uma riqueza maior de experiências com materiais e instrumentos próprios às atividades gráficas, esta idade parece que se tem evidenciado como uma fronteira pedagógica delimitante, com a conseqüente melhora nos desempenhos das crianças.

\section{CONCLUSÕES}

O estudo realizado permitiu a constatação de algumas questões que podem ser assim resumidas: as possíveis relações entre as variáveis nível socioeconômico, faixa etária e sexo e os desempenhos apresentados pelas crianças; a caracterização deste trabalho como um estudo do desenvolvimento motor; e as características do desenvolvimento da mão dominante, da população em questão.

A faixa etária foi indiscutivelmente a variável que mais se destacou, posto que os percentuais mais baixos de desempenhos constatados estavam concentrados nas crianças mais novas. Um outro aspecto que a análise da faixa etária sugere é um percentual baixo de crianças que, independente de 
sexo ou NSE, reproduziram todos os movimentos. Provavelmente isso se deve ao fato de que as circunstâncias do meio os favorecem.

O nível socioeconômico confirmou-se como a segunda variável relevante do estudo. As diversidades do meio afirmaram-se como fatores determinantes para retardar o início do desenvolvimento da mão dominante, nos movimentos destacados.

O estudo planejado em cortes transversais, na faixa etária de 3 anos e meio a 7 anos, distribuída em 8 grupos etários, em intervalos de 6 meses, permitiu uma verificação mais clara dos patamares de melhoras nos desempenhos das crianças. Se tivessem sido observadas melhoras progressivas e graduais nos desempenhos, isto poderia sugerir, entre outros aspectos, a influência prioritária de um processo de maturidade biológica. Entretanto, o que demove esta hipótese são, em primeiro lugar, as melhoras abruptas nos desempenhos das crianças do NSE baixo, a partir do momento em que passam a participar de atividades gráficas. Após 6 meses a um ano de acesso a estas atividades, o que se observa nestas crianças são desempenhos semelhant9s aos das crianças do NSE alto.

Com respeito aos movimentos reproduzidos ou não, os resultados apontam que metade (8) está sendo favorecida pelas condições de vida do cotidiano das crianças das 8 faixas etárias. Esta é uma questão que merece ser discutida junto a educadores responsáveis pelo atendimento a crianças das faixas etárias estudadas. Os movimentos da mão dominante são responsáveis pela destreza das crianças em um sem-número de tarefas. Provavelmente, a grande maioria depende da mobilidade de toda a musculatura disponível. O envolvimento das crianças em atividades que estimulem toda a musculatura da mão dominante deverá contribuir efetivamente para uma facilidade maior nas tarefas do dia-a-dia.

Quanto aos movimentos críticos para esta população, 6 dos 8 movimentos são de adução e abdução; por ordem de dificuldade, primeiro da mão, depois do indicador e por último do polegar. As atividades desta população favorecem muito mais os movimentos de extensão e flexão, com todas as partes anatômicas.

Os dados indicam, portanto, que as crianças são mais estimuladas a abrir e fechar a mão (flexão e extensão dos dedos), a abaixar e suspender a mão (flexão e extensão da mão) e a abrir e fechar os dedos (adução e abdução dos dedos). O que é relevante destacar é que elas não parecem ser estimuladas a movimentar a mão em movimentos da esquerda para a direita (adução e abdução da mão) e os dedos mais importantes para a atividade de pinça, tão necessária à escrita, como o polegar e o indicador, em movimentos da esquerda para a direita e vice-versa.

Os movimentos de flexão e extensão são os movimentos que favorecem a verticalidade dos traçados e os de abdução e adução, a horizontalidade. Assim sendo, como a escrita efetua-se nestes dois sentidos, porém num percurso de traçados fundamentalmente de sentido horizontal, os movimentos de adução e abdução configuram-se como básicos e/ou indispensáveis à mesma. Logo, parece ser necessária uma atenção especial a esses movimentos, uma vez que eles são indispensáveis a uma mobilidade maior da mão, em especial à escrita manuscrita cursiva.

Por fim, pode-se concluir que, a partir dos 3 anos e meio, algumas crianças já se mostram aptas à reprodução dos 16 movimentos básicos envolvidos na escrita manuscrita cursiva. As crianças que só evidenciaram estes desempenhos em idades posteriores parece que assim o fizeram pelas condições que lhes foram oferecidas. Estes indícios permitem sugerir que as crianças sejam liberadas para atividades gráficas criativas, a partir de seus próprios interesses, de modo a favorecer 
um progresso gradual de habilidade no controle dos movimentos da mão dominante. Sugere-se que estudos longitudinais, com maior controle do acesso aos instrumentos gráficos e outras manipulações, permitem registrar o tempo necessário ao controle na reprodução dos movimentos e seu uso efetivo na reprodução da escrita manuscrita.

\section{REFERÊNCIAS}

BIJOU, S. W.BAER, D. M. O desenvolvimento da criança. São Paulo: EPU, 1980.

BOUCHER, B. H. DOESCHER, S. M. Influencing preschool children' s motor development: a comparison of two groups. Early Child Development and Care, United Kingdom, n. 77, p. 67-76, 1991.

BUTTERWORTH, D. Gender equity in early childhood: the state of play. Australian Journal of Early Childhood, v. 16, n. 4, p. 3-9, 1991.

ENNIS, C. D. Cognitive style and gender differences in children's motor task. Early Child Development and Care, v. 64, p. 33-46,1990.

FAGOT, B. L Beyond the reinforcement principie: another step toward understanding sex role development. Development Psychology, v.21, p. 1.097-1.104, 1985.

GESELL, A. AMATRUDA, C. Diagnóstico del desarrollo normal y anormal del niño; métodos clínicos y aplicaciones prácticas. Buenos Aires: Ed. Pai dós, 1946.

HACKER, R. G. Gender studies: some methodological and theorical issues. International Journal of Science Education, v. 14, p. 527-39, 1992.

HOLLE, B. Desenvolvimento motor na criança normal e retardada; um guia prático para estimulação sensoriomotora. São Paulo: Manole, 1979.

HOL T, K. S. Developmental Paediatrics - perspectives and practice. London: Butterworths, 1977.

HURLOCK, E. B. La conducta dei nino; métodos modernos para Ia crianza durante los tres primeros anos. Buenos Aires: Soc. de Resp., 1946.

JACKLIN, C. N. Female and male: issues of gender. Journal ofthe American Psychologist Association, v.44, n.2, p. 127-33, 1989.

JONES, A GLENN, S. M. Gender differences in pretend play in a primary school group. Early Child Development and Care, v. 64, p. 61-7, 1990.

KARAPETSAS, A. VLACHOS, E. Visuomotor organization in the left-handed child; a neuropsychological approach. Perceptual and Motor Skills, v. 75, p. 699-705, 1992.

MEAD, B. J. IGNICO, A. A. A children's gender-typed perceptions of phisical activity: consequences and implications. Perceptual and Motor Skill 's, V. 75, p. 1.035-12, 1992.

SCHILLER, W. SCHILLER, J. Motor programs in early childhood training: a preservice interactive model. Early Child Development and Care, United Kingdom, v. 62, p. 49-70,1990.

SODRÉ, L. G. P. Repertório básico motor da escrita; uma proposta para seu estudo. 1982. 194 f. Dissertação (Mestrado em Educação) - Faculdade de Educação, UFBa, Salvador, 1982.

SODRÉ, L. G. P. Estudos da habilidade de escrita; identificação dos componentes gráficos envolvidos na escrita manuscrita cursiva de alfabetizadores da cidade de Salvador e a caracterização de seu padrão de escrita. Salvador: UFBa, 1985. 148 p. xerocopiado.

SODRÉ L. G. P. O desenvolvimento motor da mão dominante: um estudo descritivo com crianças da 3 anos $e$ meio a 7 anos. 1993. 370 f. Tese (Doutorado em Educação) - Faculdade de Educação, UNICAMP, Campinas, 1993.

VAUGHAN III, V. C. LITT, L F. Child and adolescent development: clinical implications. Philadelphia: W. 
B. Saunders, 1990.

WILLIAMS, J. D. Age, cohort and period in life-span research: a three-way analysis with logically missing cells. Multivariate Behavioral Research, v.26,n. 4,p. 631-54, 1991.

Recebido em 08/05/2001

Aprovado em 26/06/2001 